\title{
Electrowetting Actuation of Polydisperse Nanofluid Droplets
}

\author{
Crismar Patacsil, ${ }^{1,2}$ Jan Patrick Calupitan, ${ }^{3}$ Erwin Enriquez, ${ }^{3}$ and Raphael A. Guerrero ${ }^{1}$ \\ ${ }^{1}$ Department of Physics, Ateneo de Manila University, Loyola Heights, Quezon City, 1108 Metro Manila, Philippines \\ ${ }^{2}$ Department of Physical Sciences, University of the Philippines Baguio, Governor Pack Road, 2600 Baguio City, Philippines \\ ${ }^{3}$ Department of Chemistry, Ateneo de Manila University, Loyola Heights, Quezon City, 1108 Metro Manila, Philippines
}

Correspondence should be addressed to Raphael A. Guerrero; rguerrero@ateneo.edu

Received 10 August 2016; Revised 22 November 2016; Accepted 28 November 2016; Published 24 January 2017

Academic Editor: Peter Chang

Copyright (C) 2017 Crismar Patacsil et al. This is an open access article distributed under the Creative Commons Attribution License, which permits unrestricted use, distribution, and reproduction in any medium, provided the original work is properly cited.

\begin{abstract}
We present results of electrowetting experiments employing droplets formed from aqueous suspensions of Au nanoparticles. A planar electrowetting system, consisting of a Pt wire electrode and a bottom $\mathrm{Cu}$ electrode with an insulating silicone layer, is used to observe changes in droplet contact angle when an external electric field is applied. The equilibrium contact angle at $0 \mathrm{~V}$ decreases with increasing nanoparticle concentration, dropping from $100.4^{\circ}$ for pure deionized water to $94.7^{\circ}$ for a $0.5 \mu \mathrm{M}$ nanofluid. Increasing the nanoparticle content also lowers the required voltage for effective actuation. With actuation at $15 \mathrm{~V}$, contact angle decreases by $9 \%$ and $35 \%$ for droplets formed from pure water and a $0.5 \mu \mathrm{M}$ nanoparticle suspension, respectively. Contact angle saturation is observed with nanofluid droplets, with the threshold voltage decreasing as nanoparticle concentration rises. Maximum droplet actuation before contact angle saturation is achieved at only $10 \mathrm{~V}$ for a concentration of $0.5 \mu \mathrm{M}$. A proposed mechanism for the enhanced electrowetting response of a nanofluid droplet involves a reduction in surface tension of the droplet as nanoparticles accumulate at the liquid-vapor interface.
\end{abstract}

\section{Introduction}

Numerous applications involving the actuation of fluids at miniaturized scales are enabled by the variable wettability of contact surfaces. In electrowetting (EW), an established method for fluid manipulation, an applied electric field affects surface tension and modifies how a liquid spreads over a substrate [1-4]. With EW methods, wettability of a surface is modified without changing the chemical composition of the liquid or substrate. Applications of EW in microfluidic systems range from laboratory-on-a-chip platforms for the preparation and analysis of biological samples [5-7] to color displays [8-10]. The basic design of a liquid droplet in contact with an appropriate substrate avoids issues involving microfabrication, with electrowetting on dielectric (EWOD) droplet systems performing as easily deformable optical elements that are cost-effective and disposable [11-13]. EWOD is currently the most common actuation method in digital microfluidic architectures [14]. In this paper, we report the use of a planar EWOD set-up to actuate nanofluid droplets.
Nanofluids refer to liquid elements containing suspensions of nanoparticles or other nanosized structures $[15,16]$. In electrowetting experiments, a nanofluid droplet exhibits an altered contact angle response due to the presence of suspended nanoparticles. For droplets containing submicron polystyrene beads [15], both particle size and volume fraction had an effect on the equilibrium contact angle. In addition, contact angle saturation was observed at a much higher voltage compared to the saturation voltage of a conventional water droplet. EWOD actuation of droplets formed from aqueous suspensions of bismuth telluride nanoparticles revealed that nanoparticles enhanced the mobility of charge transport [16]. As a result of large conductivity, the nanofluid did not exhibit contact angle saturation within an actuation range of 0 to $60 \mathrm{~V}$.

For EWOD experiments reported here, we employ droplets of aqueous solutions of Au nanoparticles (AuNPs). The nanoparticles range in diameter from 5 to $40 \mathrm{~nm}$. Preparation of the nanofluid is accomplished via a simple synthesis method that may be completed within a few minutes [17]. 
Based on the available literature, the EW performance of this specific type of nanofluid has yet to be evaluated. To the best of our knowledge, we are the first to report experimental results of EWOD actuation of aqueous suspensions of polydisperse AuNPs. AuNPs are known to possess material properties that are useful in catalysis protocols and biomedicine [18]. Due to excellent biocompatibility [19], AuNPs are being considered for applications in biomedical diagnostics and photodynamic therapies [20]. Studies on EW actuation of nanoparticle suspensions are motivated by the potential utility of nanofluids in microscale fluidic systems. The unique properties of nanofluids may find applications in microchannel cooling of electronic circuits and the lubrication of manufacturing processes [16]. Our EWOD system for a nanofluid droplet may serve as a platform for fluid sensing [12]. EW of nanofluids could also be a component of studies focusing on the transport of biological macromolecules [15]. Plasmon resonance excitation [21], a phenomenon that leads to interesting optical properties of spherical nanoparticles, is not considered in this present work.

Our paper is structured as follows: Section 2 discusses details of the AuNP synthesis involved in preparing nanofluid samples. The EW set-up for contact angle measurements is described in Section 3. We present our results for EW experiments in Section 4 and provide a simple analysis of the observed EW response in Section 5. Section 6 contains our concluding remarks.

\section{Nanofluid Preparation Method}

Synthesis of AuNPs was accomplished through a protocol first described in [17]. Preparation of the nanofluid takes less than 10 minutes and does not require a cleaning step. The main process involves the reduction of $\mathrm{Au}$ (III) ions to $\mathrm{Au}$ in water, effecting the formation of negatively charged AuNPs that are stable via electrostatic repulsion due to adsorbed anions. To prepare a $0.5 \mu \mathrm{M}$ suspension of AuNPs, we started with a base solution of $\mathrm{HAuCl}_{4}$ diluted with deionized water. Upon adding the reductant $\mathrm{NaBH}_{4}$ to the $\mathrm{HAuCl}_{4}$ solution, the fluid immediately turned orange and then brown. After four minutes of stirring at room temperature, the solution turned red, a color corresponding to the surface plasmon resonance of AuNPs. Additional molar concentrations were made available by adding deionized water. We produced solutions with the following concentrations: $0.5,0.33,0.25$, and $0.05 \mu \mathrm{M}$.

The presence of AuNPs was confirmed by UV-Vis spectroscopy and with an atomic force microscope (AFM). Figure 1 is the absorbance spectrum of the $0.5 \mu \mathrm{M}$ AuNP solution for the wavelength range of 350 to $750 \mathrm{~nm}$. Polydisperse AuNPs typically exhibit a broad absorption band [18]. Peak absorbance of our nanofluid sample is observed at $515 \mathrm{~nm}$, corresponding to an average nanoparticle diameter of less than $10 \mathrm{~nm}$ [22]. To facilitate AFM imaging, the dropcasting method was used to deposit an amount of the AuNP suspension onto an arbitrary substrate. The atomic force micrograph in Figure 2 confirms the wide size distribution of the AuNPs. Within the representative viewing area of

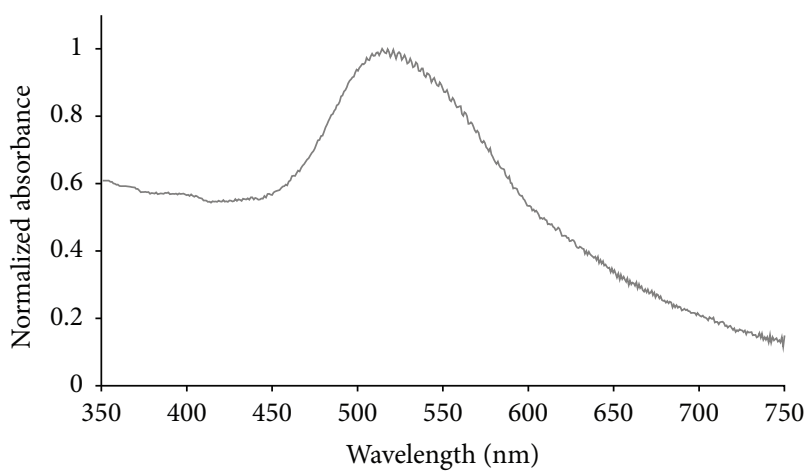

Figure 1: Absorbance spectrum of a $0.5 \mu \mathrm{M}$ AuNP suspension. The peak at $515 \mathrm{~nm}$ corresponds to an average nanoparticle diameter of approximately $10 \mathrm{~nm}$.

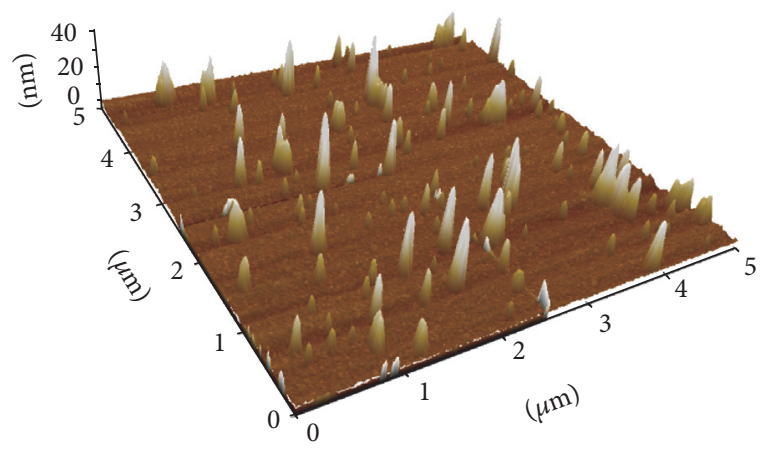

FIgURE 2: Synthesized AuNPs on an arbitrary substrate imaged via atomic force microscopy.

$5 \times 5 \mu \mathrm{m}^{2}$, nanoparticle diameters are seen to range from 5 to $40 \mathrm{~nm}$. In the original report detailing the AuNP synthesis method employed in this study, Au nanoparticles ranging in diameter from 3.2 to $5.2 \mathrm{~nm}$ were produced [17]. Our average particle diameter of $\sim 10 \mathrm{~nm}$ is of comparable scale. The discrepancy in particle size distribution resulting from similar preparation protocols may be attributed to AuNP agglomeration during the application of nanofluid on the substrate.

\section{Electrowetting System Details}

In a standard EW system, a conducting liquid rests on a solid electrode with a thin insulating layer separating the two components [1]. Figure 3 is a schematic diagram of our EW system for the actuation of nanofluid droplets. A conducting thin film deposited on a $25 \times 25 \mathrm{~mm}^{2}$ glass slide serves as the bottom electrode in our planar EW set-up. To fabricate the thin film, we perform thermal vapor deposition of $\mathrm{Cu}$ at a base pressure of $2.6 \times 10^{-5}$ Torr. We are able to produce a conducting film with thickness of $\sim 70 \mathrm{~nm}$ after a deposition time of $60 \mathrm{~s}$. The surface of the electrode is spincoated with a polydimethylsilicone (PDMS) elastomer base (Sylgard 184) at $6000 \mathrm{rpm}$ for $300 \mathrm{~s}$. No curing agent is mixed with the elastomer and the PDMS layer remains in liquid form. Our current set-up does not allow direct measurement of the resulting PDMS layer thickness. A Pt wire electrode 


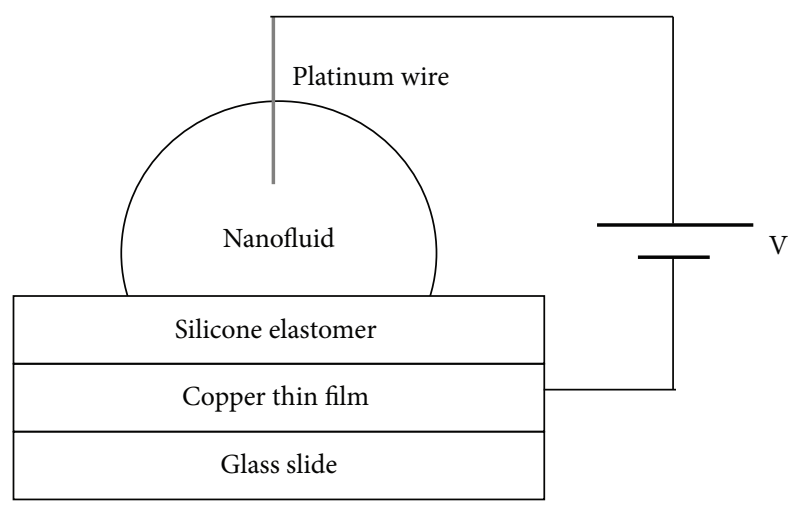

FIGURE 3: Schematic diagram of a planar electrowetting set-up for the actuation of a nanofluid droplet.

(diameter $=255 \mu \mathrm{m}$ ) is inserted at the top of the droplet for the application of DC voltage. We mount the compact EW assembly on a custom translation stage to facilitate contact angle measurements. A similar EW set-up was employed in demonstrating a color display based on a fluorescent droplet [23]. The substrate used in the droplet display did not have a spin-coated dielectric layer and the actuated fluid was a laser dye solution.

We use a digital microscope to capture images for determining contact angles. The microscope is equipped with white LEDs for illuminating samples. Curvature profiles are obtained with the droplet in front of a white background. The resulting high contrast images are processed using ImageJ software [24].

\section{Experimental Results}

Deionized water is used as a testing liquid for the electrowetting set-up. Figures 4(a) and 4(b) illustrate how the wetting behavior of a water droplet is modified by an applied electric field. The droplet exhibits a contact angle of $100.4^{\circ}$ at $0 \mathrm{~V}$. Contact angle decreases with voltage, dropping to $96^{\circ}$ at an actuation voltage of $10 \mathrm{~V}$. Along with a change in droplet curvature, the base of the fluid increases in area by $29 \%$ due to spreading induced by EW. Spreading of the droplet is attributed to a normal force, acting on the fluid surface near the edge, generated by an electric field due to excess charges distributed on the liquid surface [2]. In Figures 4(c) and 4(d), we replace the water droplet with a droplet sourced from our $0.5 \mu \mathrm{M}$ AuNP solution. Enhanced EW behavior is observed with a nanofluid droplet. Beginning with a lower value of $94.7^{\circ}$, contact angle decreases by almost $30 \%$ to $67.2^{\circ}$ with a low actuation voltage of $10 \mathrm{~V}$. The droplet base area becomes $50 \%$ larger at this level of actuation.

Figure 5 plots the range of contact angles accessible with the nanofluid droplet under EW actuation as a function of voltage. The EW response of various concentrations of AuNP suspensions is compared with that of deionized water. With no applied voltage, the unactuated contact angle assumes smaller values with AuNP concentration. From a value of $100.4^{\circ}$ for deionized water, the initial contact angle becomes $98.9^{\circ}$ for a nanofluid with the lowest concentration of $0.005 \mu \mathrm{M}$, eventually reaching $94.7^{\circ}$ for a $0.5 \mu \mathrm{M}$ droplet. We relate the altered equilibrium contact angle to a decrease in liquid-vapor surface tension due to the presence of AuNPs [15]. Droplet actuation becomes more pronounced at higher AuNP concentrations. For concentrations of 0.05, 0.25, 0.33, and $0.5 \mu \mathrm{M}$, the contact angles measured at $15 \mathrm{~V}$ are $85.9^{\circ}$, $77.6^{\circ}, 67.5^{\circ}$, and $61.9^{\circ}$, respectively. Contact angle of the deionized water droplet at the same applied voltage is $91^{\circ}$. Modified contact angles decrease by $10.3 \%$ between concentration steps on average. Due to the onset of saturation effects [1], the reduction in contact angle at successive concentration levels drops to $1.7 \%$ for actuation at $30 \mathrm{~V}$, with contact angles of $59^{\circ}, 58.4^{\circ}, 58.5^{\circ}$, and $55.9^{\circ}$, respectively, for the same levels of increasing concentration. At this maximum actuation voltage, we measure a contact angle of $68^{\circ}$ for deionized water. For both cases of applied voltage, the actuated angles achieved with nanofluid droplets are significantly smaller compared to those observed with droplets of deionized water.

Voltage requirements for droplet actuation become lower as AuNP concentration rises. At $15 \mathrm{~V}$, contact angle for a water droplet decreases by only $9 \%$. With the same applied voltage, the nanofluid contact angle changes by $13 \%, 19 \%, 30 \%$, and $35 \%$ for $0.05,0.25,0.33$, and $0.5 \mu \mathrm{M}$ droplets, respectively. We are able to significantly modify the wetting capability of our electrode surface with minimal applied voltage. EW behavior follows the expected parabolic relationship between the cosine of the contact angle and voltage [11], seen in Figure 5 as solid lines. Contact angle saturation is not observed with the deionized water droplet but is evident with our nanofluid droplets. Deviation from the expected EW trend occurs at $26 \mathrm{~V}$ for the lowest AuNP concentration. At 0.25, 0.33, and $0.5 \mu \mathrm{M}$ concentrations, the contact angle saturates at 19,16 , and $10 \mathrm{~V}$, respectively. Contact angle saturation in this case is due to a reduction of EW force resulting from trapped charge in the insulating layer [1]. Saturation may also be a consequence of low electrical conductivity of the solution [16]. The presence of AuNPs in low concentrations does not appear to modify limiting factors of droplet spreading, such as the amount of charge adsorbed at the liquid-solid interface [4]. Within our applied voltage range, the maximum variation in contact angle with respect to the unactuated value is approximately $40 \%$ for all AuNP concentrations. On average, the contact angle saturates at $65.4^{\circ}$, with all concentrations producing angles within $2.8 \%$ of this mean value. A thermodynamic limit of stability accounted for similar saturation behavior at $65^{\circ}$ with an applied voltage of $200 \mathrm{~V}$ [25]. Our results for the AuNP nanofluid represent an improvement over the $23 \%$ decrease in contact angle reported for suspensions of bismuth telluride nanoparticles at a larger applied voltage of $60 \mathrm{~V}$ [16].

\section{Discussion}

The Young-Lippmann equation provides an expression for the variation in contact angle $\theta$ as a function of applied voltage $V$ :

$$
\cos \theta=\cos \theta_{o}+\chi_{\phi} V^{2}
$$




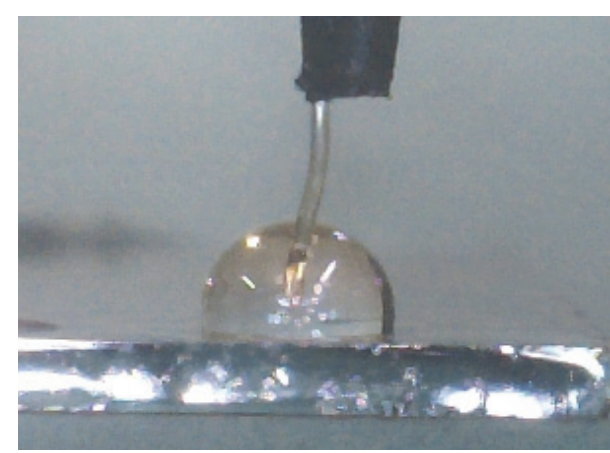

(a)

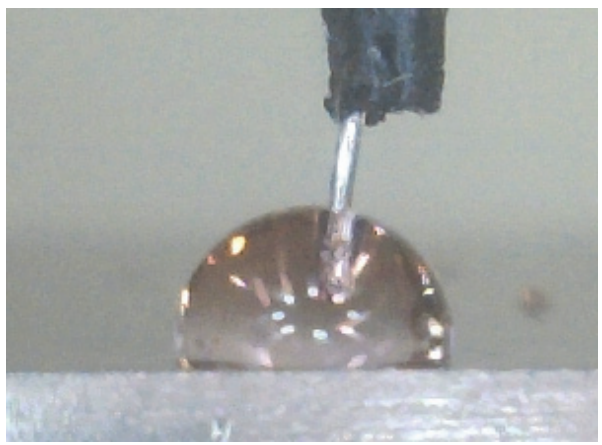

(c)

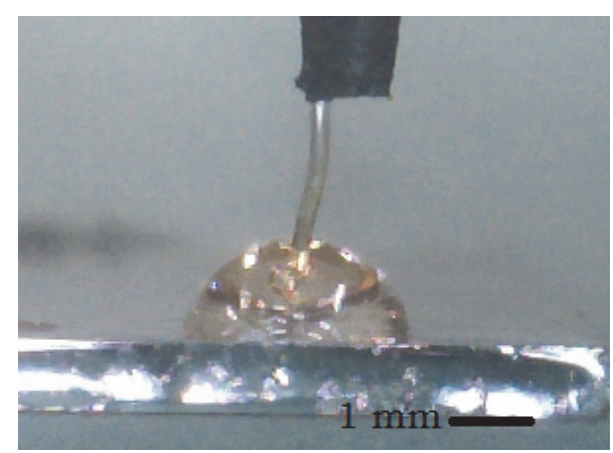

(b)

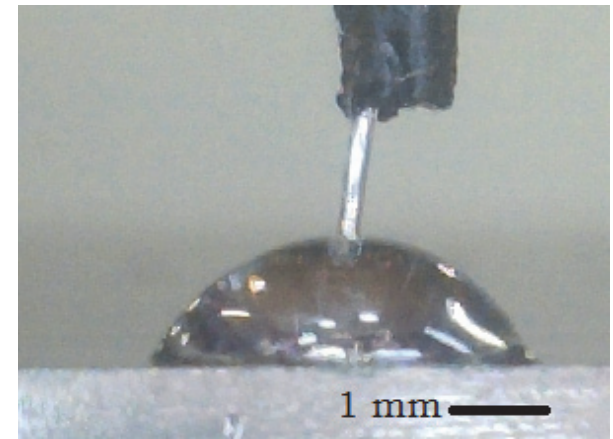

(d)

Figure 4: Change in droplet curvature and base diameter due to electrowetting actuation for deionized water: (a) at $0 \mathrm{~V}$; (b) at $10 \mathrm{~V}$. For a $0.5 \mu \mathrm{m}$ nanofluid droplet, (c) a smaller contact angle at $0 \mathrm{~V}$ and (d) an enhanced electrowetting response at $10 \mathrm{~V}$ are observed.

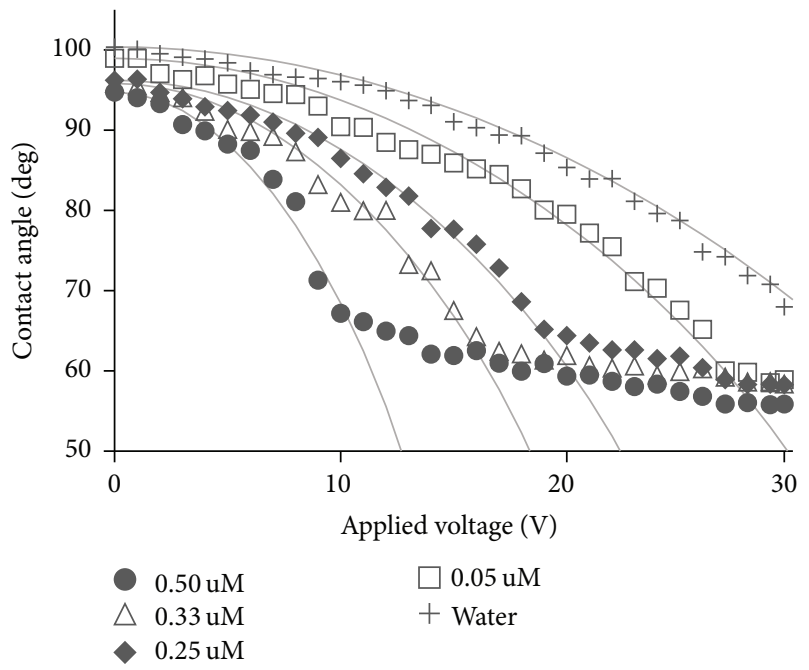

FIGURE 5: Contact angle versus applied voltage for droplets of different AuNP concentrations. The solid lines represent the expected quadratic dependence of contact angle on voltage. Higher concentrations reduce voltage requirements for effective actuation.

where the equilibrium angle $\theta_{o}$ depends on surface tensions at the liquid-vapor, solid-liquid, and solid-vapor interfaces $[7,15]$. To accommodate the presence of nanoparticles in the fluid, we introduce a modified linearity coefficient $\chi_{\phi}$ defined as

$$
\chi_{\phi}=\frac{1}{2 \sigma_{\mathrm{lv}}(\phi)}\left(C_{o}^{\prime}+\Delta C^{\prime}(\phi)\right) .
$$

$C_{o}^{\prime}$ is the effective capacitance per unit area:

$$
C_{o}^{\prime}=\frac{\epsilon_{o} \epsilon_{r}}{d}
$$

where $\epsilon_{o}$ is the vacuum permittivity, $\epsilon_{r}$ is the relative permittivity, and $d$ is the dielectric layer thickness. Effects of nanoparticle concentration $\phi$ include a change in effective capacitance $\Delta C^{\prime}(\phi)$ which vanishes at $\phi=0$. Particles in the fluid act as a suspended dielectric and increase the electrical permittivity [15]. Nanofluid surface tension $\sigma_{\mathrm{lv}}(\phi)$ at the liquid-vapor interface is also affected by the presence of nanoparticles. Electrostatic repulsion among nanoparticles accumulating at the interface with rising concentration reduces fluid surface energy [26]. For low levels of $\phi$, surface tension weakens as concentration increases. The drop in surface tension is consistent with our observation of smaller equilibrium contact angles at higher concentrations. From the Young-Lippmann equation, an increasing trend for $\chi_{\phi}$ would account for lower voltage requirements when actuating nanofluids with more AuNP content.

From contact angle data in Figure $5, \chi_{\phi}$ has a value of $0.0006 \mathrm{~V}^{-2}$ for a pure water droplet. Applying (2) and (3), with $\epsilon_{r}=2.5$ for PDMS and $\sigma_{\mathrm{lv}}=0.072 \mathrm{~N} / \mathrm{m}$ for water $[15,22]$, we calculate the dielectric layer thickness $d$ to be $0.26 \mu \mathrm{m}$. Resulting $C_{o}^{\prime}$ for the same droplet is $8.5 \times 10^{-5} \mathrm{~F} / \mathrm{m}^{2}$. The theoretical curves in Figure 5 are based on (1) with the linearity coefficient $\chi_{\phi}$ calibrated to best fit each experimental data set. Nanoparticles enhance the dielectric constant of the composite droplet, although particle concentration contributes more to the modification of interfacial areas at 


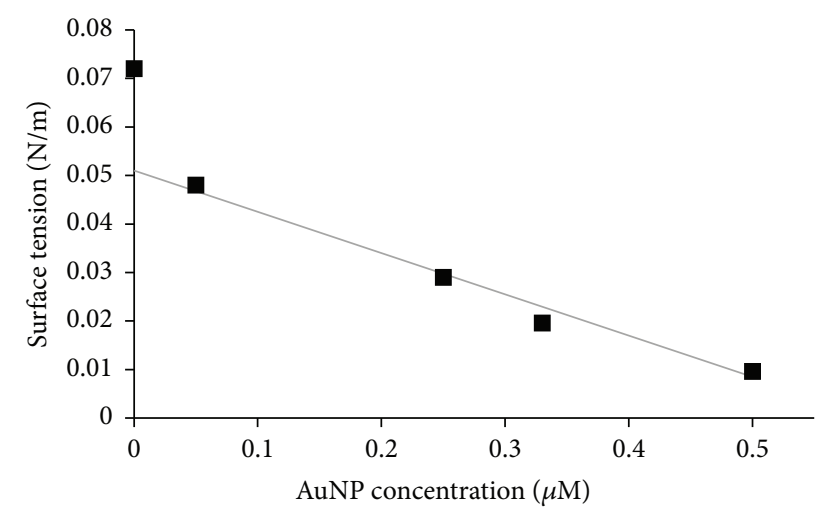

FIGURE 6: Liquid-vapor surface tension versus AuNP concentration. Surface energy decreases linearly with concentration as nanoparticles accumulate at the liquid-vapor interface.

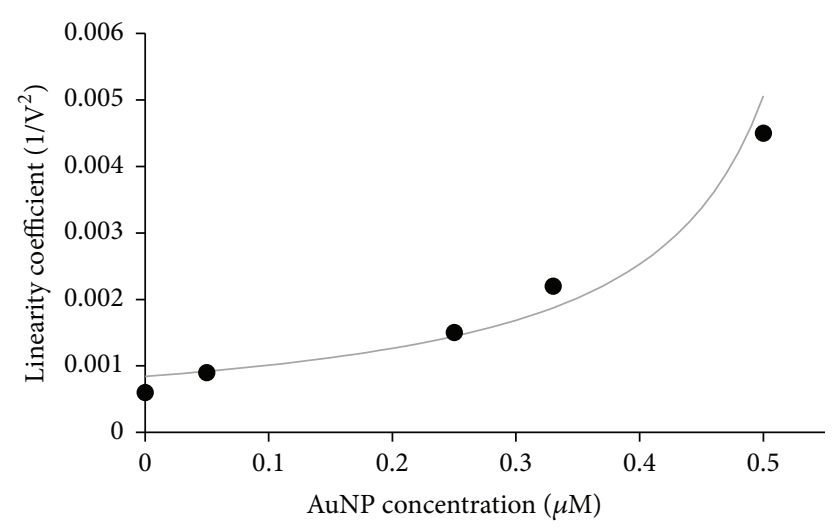

FIGURE 7: Linearity coefficient in the Young-Lippmann equation as a function of AuNP concentration. The theoretical curve is based on surface tension weakening as nanoparticle content rises.

low voltages [15]. Given the difference in order of magnitude between $\sigma_{\mathrm{lv}}$ and $C_{o}^{\prime}$ for water, as well as the level of our applied voltages, we take $\sigma_{\mathrm{lv}}(\phi)$ to be the dominant factor in (2). We plot $\sigma_{\mathrm{lv}}(\phi)$ as a function of AuNP concentration in Figure 6, applying the approximation that $\Delta C^{\prime}(\phi)$ is negligible. With a $0.05 \mu \mathrm{M}$ droplet, $\sigma_{\mathrm{lv}}(\phi)$ decreases by $33 \%$ to $0.048 \mathrm{~N} / \mathrm{m}$. The drop in surface tension is comparable with results obtained with bismuth telluride nanoparticles in deionized water, where a reduction in surface energy is caused by the accumulation of particles at the liquid-vapor interface [26]. In decreasing to $0.0096 \mathrm{~N} / \mathrm{m}$ at the highest concentration of $0.5 \mu \mathrm{M}, \sigma_{\mathrm{lv}}(\phi)$ follows a linear dependence on $\phi$. The empirical fit (solid line) is based on

$$
\sigma_{\mathrm{lv}}(\phi)=\sigma_{\mathrm{lv}}(0)-\alpha \phi,
$$

where $\sigma_{\mathrm{lv}}(0)$ is the surface tension of a pure water droplet. For the range of AuNP concentrations considered in this study, (4) accurately describes $\sigma_{\mathrm{lv}}(\phi)$ with slope $\alpha=8.5 \times$ $10^{4} \mathrm{~N} /(\mathrm{mol} \cdot \mathrm{m})$. The transition from $\sigma_{\mathrm{lv}}(0)$ and the approach to a limiting nonzero value for surface tension are not included in this simple linear treatment.

$\chi_{\phi}$ values extracted from our EW data are plotted in Figure 7 as a function of AuNP concentration. As expected, within the concentration range of our experiments, $\chi_{\phi}$ consistently increases with $\phi$. The solid line included in the plot is a theoretical curve calculated from (2), with $\sigma_{\mathrm{lv}}(\phi)$ decreasing linearly with AuNP concentration as in (4). We maintain the approximation that $\Delta C^{\prime}(\phi) \ll C_{o}^{\prime}$. Experimental linearity coefficients are in excellent agreement with theory. Our treatment of the decrease in liquid-vapor surface tension due to the presence of AuNPs as the dominant factor in modifying EW response is valid within this concentration range. Deviations from the expected behavior may be due to effects involving charge transport at the liquid-dielectric interface and modified capacitance of the nanofluid droplet [16]. In addition, the liquid-vapor surface tension may be approaching a minimum value at higher AuNP concentrations.

Our technique allows for significant droplet actuation at minimal applied voltages. At maximum $\phi$, EW actuation of our nanofluid occurs with $\chi_{\phi}=0.0045 \mathrm{~V}^{-2}$. A study on EW of suspensions of submicron polystyrene beads reported a coefficient three orders of magnitude smaller [15]. Different experimental conditions account for this disparity in measured values, with the linearity coefficient depending on nanoparticle type and EW system parameters such as the choice of dielectric layer and its prepared thickness.

\section{Conclusion}

In summary, we report effective EW actuation of droplets formed from aqueous suspensions of polydisperse AuNPs at low voltages. Smaller actuation voltages are required for nanofluids with larger nanoparticle concentrations, within the regime of low molarity suspensions. The enhanced EW response is attributed to reduced surface tension of the nanofluid when nanoparticle concentration is elevated.

A polydisperse nanofluid containing AuNPs exhibits great potential as the active liquid in EW systems due to low voltage requirements and a tunable actuation response based on concentration. Our EW set-up for a nanofluid droplet may be readily modified to serve as a versatile microfluidic platform for miniaturized imaging devices and on-chip spectroscopy schemes. The actuation properties of AuNP suspensions could find utility in studying surface dynamics of solid-liquid interfaces.

\section{Competing Interests}

The authors declare that there are no competing interests regarding the publication of this paper.

\section{Acknowledgments}

The authors thank R. D. Mero for her input during their initial electrowetting experiments and M. P. Jallorina for his assistance in preparing their $\mathrm{Cu}$ electrodes. Crismar Patacsil acknowledges support from the Accelerated Science and Technology Human Resource Development Program and the University of the Philippines Doctoral Studies Program. Equipment grants were provided by the Philippine Council 
for Industry, Energy and Emerging Technology Research and Development.

\section{References}

[1] H. J. J. Verheijen and M. W. J. Prins, "Reversible electrowetting and trapping of charge: model and experiments," Langmuir, vol. 15, no. 20, pp. 6616-6620, 1999.

[2] K. H. Kang, "How electrostatic fields change contact angle in electrowetting," Langmuir, vol. 18, no. 26, pp. 10318-10322, 2002.

[3] B. P. Cahill, A. T. Giannitsis, R. Land et al., "Reversible electrowetting on silanized silicon nitride," Sensors and Actuators, B: Chemical, vol. 144, no. 2, pp. 380-386, 2010.

[4] M. Vallet, M. Vallade, and B. Berge, "Limiting phenomena for the spreading of water on polymer films by electrowetting," European Physical Journal B, vol. 11, no. 4, pp. 583-591, 1999.

[5] Y.-P. Zhao and Y. Wang, "Fundamentals and applications of electrowetting: a critical review," Reviews of Adhesion and Adhesives, vol. 1, no. 1, pp. 114-174, 2013.

[6] Y. S. Nanayakkara, H. Moon, T. Payagala et al., "A fundamental study on electrowetting by traditional and multifunctional ionic liquids: possible use in electrowetting on dielectric-based microfluidic applications," Analytical Chemistry, vol. 80, no. 20, pp. 7690-7698, 2008.

[7] R. Shamai, D. Andelman, B. Berge, and R. Hayes, "Water, electricity, and between... On electrowetting and its applications," Soft Matter, vol. 4, no. 1, pp. 38-45, 2007.

[8] R. Massard, J. Mans, A. Adityaputra, R. Leguijt, C. Staats, and A. Giraldo, "Colored oil for electrowetting displays," Journal of Information Display, vol. 14, no. 1, pp. 1-6, 2013.

[9] R. A. Hayes and B. J. Feenstra, "Video-speed electronic paper based on electrowetting," Nature, vol. 425, no. 6956, pp. 383385, 2003.

[10] H. You and A. J. Steckl, "Three-color electrowetting display device for electronic paper," Applied Physics Letters, vol. 97, no. 2, Article ID 023514, 2010.

[11] A. Kiraz, Y. Karadag, and A. F. Coskun, "Spectral tuning of liquid microdroplets standing on a superhydrophobic surface using electrowetting," Applied Physics Letters, vol. 92, no. 19, Article ID 191104, 2008.

[12] A. Kiraz, S. Ç. Yavuz, Y. Karadağ, A. Kurt, A. Sennaroglu, and H. Çankaya, "Large spectral tuning of liquid microdroplets standing on a superhydrophobic surface using optical scattering force," Applied Physics Letters, vol. 91, no. 23, Article ID 231102, 2007.

[13] A. Kiraz, M. A. Dündar, A. L. Demirel et al., "Single glycerol/water microdroplets standing on a superhydrophobic surface: optical microcavities promising original applications," Journal of Nanophotonics, vol. 1, no. 1, Article ID 011655, 2007.

[14] L. Mats, A. Bramwell, J. Dupont, G. Liu, and R. Oleschuk, "Electrowetting on superhydrophobic natural (Colocasia) and synthetic surfaces based upon fluorinated silica nanoparticles," Microelectronic Engineering, vol. 148, pp. 91-97, 2015.

[15] D. Chakraborty, G. S. Sudha, S. Chakraborty, and S. DasGupta, "Effect of submicron particles on electrowetting on dielectrics (EWOD) of sessile droplets," Journal of Colloid and Interface Science, vol. 363, no. 2, pp. 640-645, 2011.

[16] R. K. Dash, T. Borca-Tasciuc, A. Purkayastha, and G. Ramanath, "Electrowetting on dielectric-actuation of microdroplets of aqueous bismuth telluride nanoparticle suspensions," Nanotechnology, vol. 18, no. 47, Article ID 475711, 2007.
[17] M. N. Martin, J. I. Basham, P. Chando, and S.-K. Eah, "Charged gold nanoparticles in non-polar solvents: 10-min synthesis and 2D self-assembly," Langmuir, vol. 26, no. 10, pp. 7410-7417, 2010.

[18] H. Qian, M. Zhu, E. Lanni, Y. Zhu, M. E. Bier, and R. Jin, "Conversion of polydisperse Au nanoparticles into monodisperse $\mathrm{Au}_{25}$ nanorods and nanospheres," The Journal of Physical Chemistry C, vol. 113, no. 41, pp. 17599-17603, 2009.

[19] M. Shah, V. D. Badwaik, and R. Dakshinamurthy, "Biological applications of gold nanoparticles," Journal of Nanoscience and Nanotechnology, vol. 14, no. 1, pp. 344-362, 2014.

[20] L. A. Dykman and N. G. Khlebtsov, "Gold nanoparticles in biology and medicine: recent advances and prospects," Acta Naturae, vol. 3, no. 2, pp. 34-55, 2011.

[21] K. L. Kelly, E. Coronado, L. L. Zhao, and G. C. Schatz, "The optical properties of metal nanoparticles: the influence of size, shape, and dielectric environment," Journal of Physical Chemistry B, vol. 107, no. 3, pp. 668-677, 2003.

[22] M.-C. Daniel and D. Astruc, "Gold nanoparticles: assembly, supramolecular chemistry, quantum-size-related properties, and applications toward biology, catalysis, and nanotechnology," Chemical Reviews, vol. 104, no. 1, pp. 293-346, 2004.

[23] R. A. Guerrero and R. D. C. Mero, "Electrowetting actuation of a dye-doped fluorescent droplet," in Nanophotonics and Micro/Nano Optics II, vol. 9277 of Proceedings of SPIE, Beijing, China, October 2014.

[24] C. A. Schneider, W. S. Rasband, and K. W. Eliceiri, "NIH Image to ImageJ: 25 years of image analysis," Nature Methods, vol. 9, no. 7, pp. 671-675, 2012.

[25] A. Quinn, R. Sedev, and J. Ralston, "Contact angle saturation in electrowetting," Journal of Physical Chemistry B, vol. 109, no. 13, pp. 6268-6275, 2005.

[26] S. Vafaei, A. Purkayastha, A. Jain, G. Ramanath, and T. BorcaTasciuc, "The effect of nanoparticles on the liquid-gas surface tension of $\mathrm{Bi}_{2} \mathrm{Te}_{3}$ nanofluids," Nanotechnology, vol. 20, no. 18, Article ID 185702, 2009. 

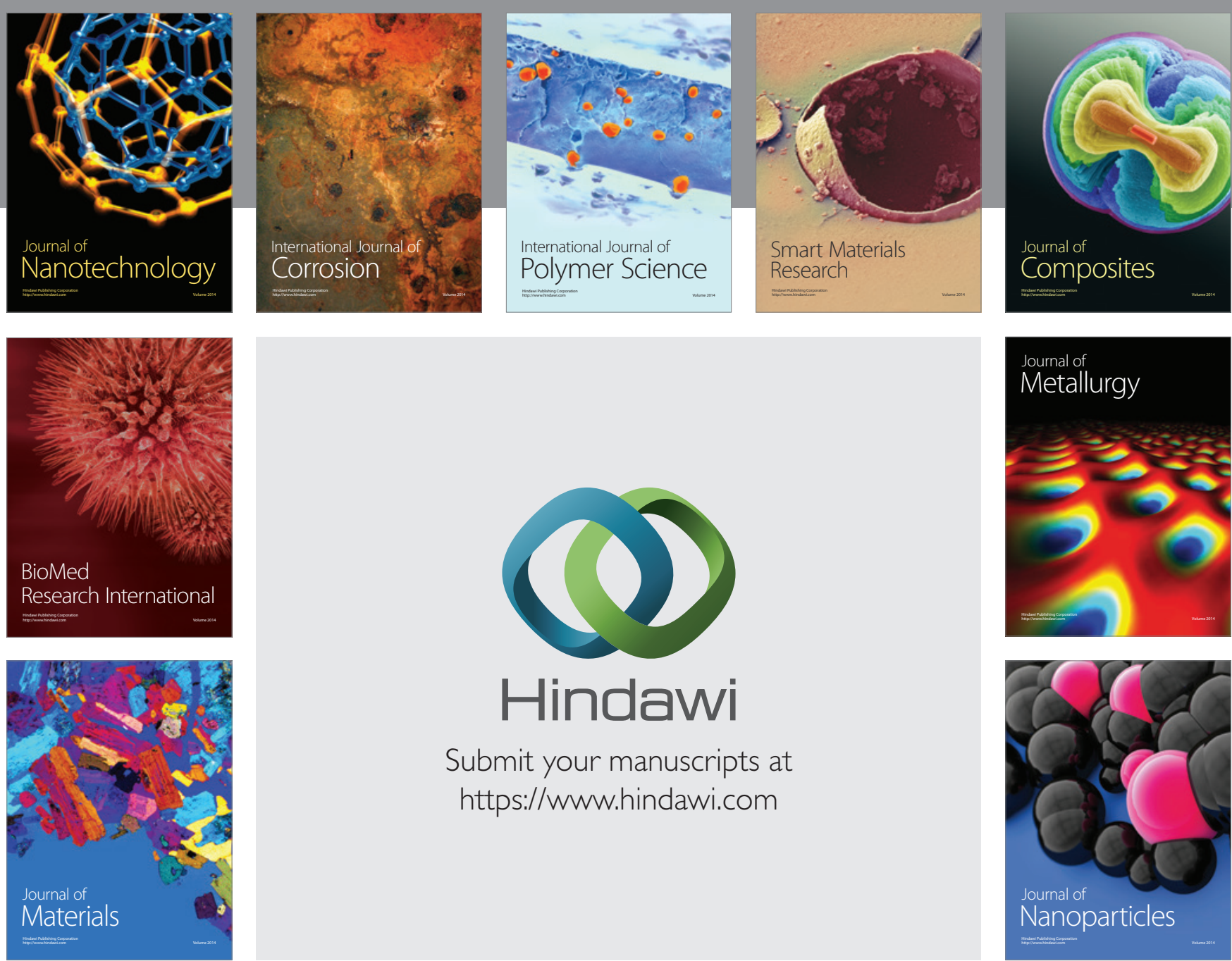

\section{Hindawi}

Submit your manuscripts at

https://www.hindawi.com

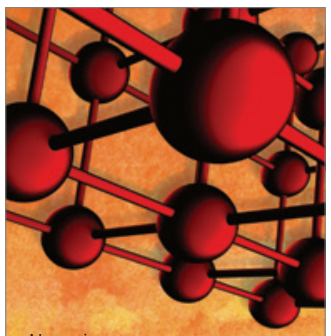

Materials Science and Engineering
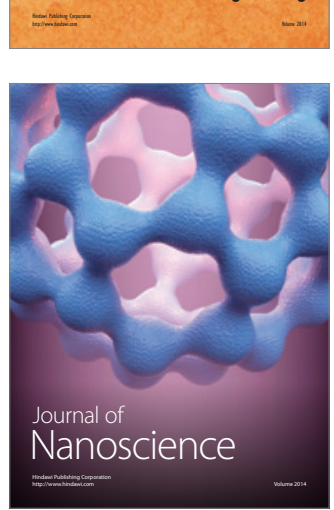
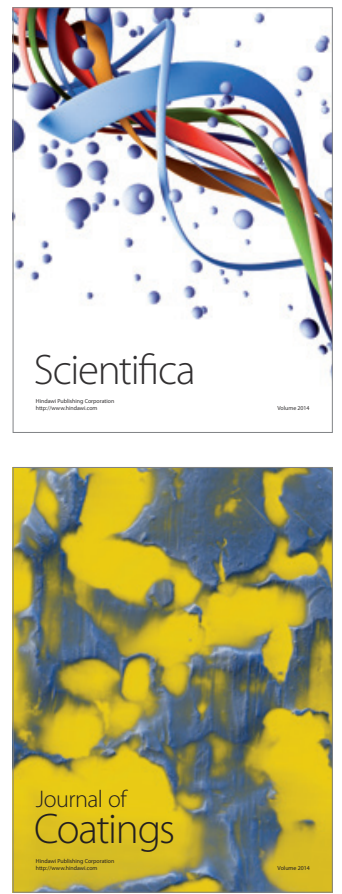
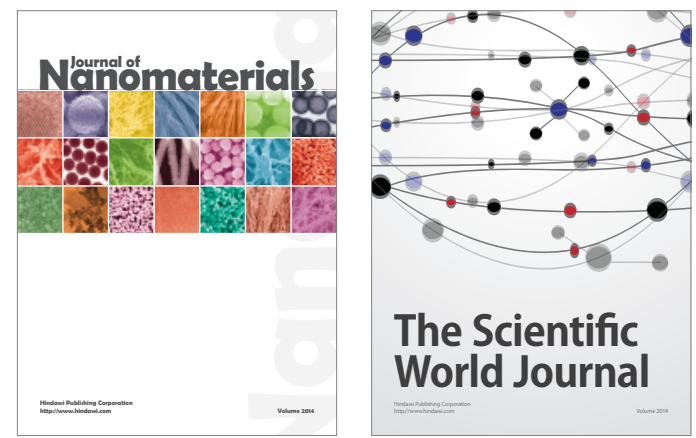

The Scientific World Journal
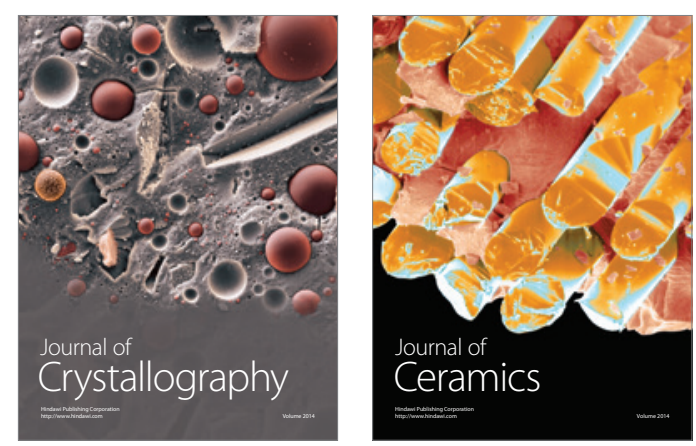
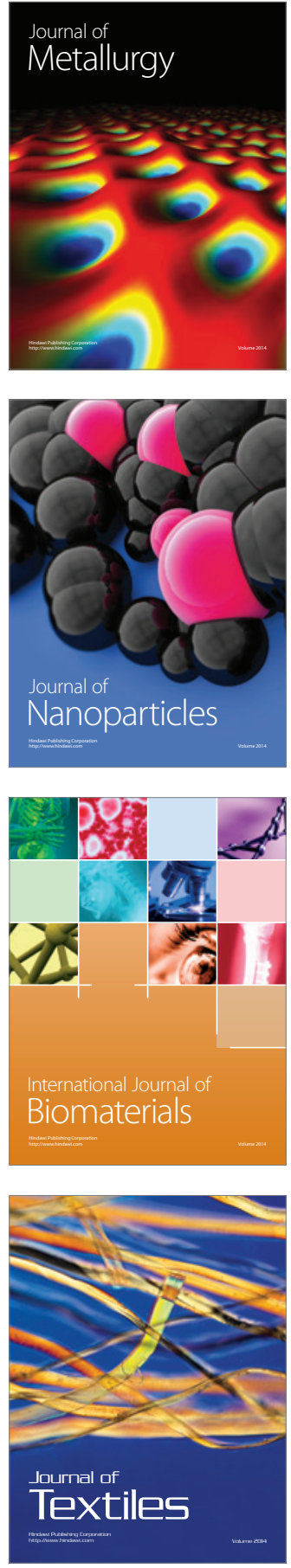